\title{
‥
}

CLINICAL RESEARCH

\section{Assessment of treatment outcomes for facial prostheses in patients with craniofacial defects: A pilot retrospective study}

\author{
Thais Bianca Brandão, DDS, MSc, ${ }^{a}$ Aljomar José Vechiato Filho, DDS, MSc, ${ }^{b}$
}

Victor Eduardo de Souza Batista, DDS, MSc, ${ }^{c}$ Ana Carolina Prado Ribeiro, DDS, MSc, PhD, ${ }^{d}$

Hugo Nary Filho, DDS, MSc, PhD, ${ }^{e}$ Israel Chilvarquer, DDS, MSc, PhD, ${ }^{f}$ Martha E. Nunn, DDS, PhD, ${ }^{g}$ Alan Roger Santos-Silva, DDS, MSc, PhD, ${ }^{\text {h }}$ Valentim Adelino Ricardo Barão, DDS, MSc, PhD, ${ }^{\text {, and }}$

Alvin G. Wee, DDS, PhD, MPH

\begin{abstract}
Statement of problem. The longevity of silicone facial prostheses is short, and published data concerning this type of rehabilitation are limited.

Purpose. The purpose of this retrospective study was to identify predictive variables for prosthetic failure and to highlight the results that can be expected after treatment with silicone facial prostheses.

Material and methods. After institutional approval, patient records from a single Brazilian institution for the time period 2004 to 2015 were assessed. A standardized form was used to collect patient data. Frailty survival modeling (simple random effects survival model) was used to test whether age, sex, type of prosthesis, source of defect, number of implants, and type of retention have a direct impact on the prosthetic failure rate $(\alpha=.05)$.
\end{abstract}

Results. Auricular prostheses were the most frequently fabricated prostheses. Eighty-four prostheses were fabricated during the follow-up period. The overall survival rate for facial prostheses was $34.5 \%$. Color alteration was the most common reason for new prostheses (27.38\%). The implant success rate was $98.18 \%$. The number of implants approached statistical significance $(P=.06)$ with a reduced risk of failure when the patient had 2 implants compared with patients with zero implants and patients with more than 2 implants.

Conclusions. Patients should be recalled frequently so that retouches can be performed, avoiding the repeated fabrication of new prostheses. Well-designed studies are necessary to identify more relevant complications and factors that lead to prosthetic failure. (J Prosthet Dent 2017;118:235-241)

Silicone facial prostheses have been proposed as the best approach to rehabilitating patients with craniofacial defects. ${ }^{1-3}$ However, the longevity and maintenance of facial prostheses are the principal concerns of clinicians and patients who undergo this method of rehabilitation. ${ }^{1,3,4}$ Prosthesis retention, which is assumed to be the primary determinant of a successful prosthetic approach, has traditionally been achieved using adhesives or

\footnotetext{
Supported by the Brånemark Institute and Nobel Biocare.

${ }^{a}$ Coordinator, Dental Oncology Service, Institute of Cancer of São Paulo (ICESP), São Paulo, Brazil; and Coordinator, Anaplastology Service of Brånemark Institute, São Paulo, Brazil.

${ }^{\text {b} A s s i s t a n t, ~ D e n t a l ~ O n c o l o g y ~ S e r v i c e, ~ I n s t i t u t e ~ o f ~ C a n c e r ~ o f ~ S a ̃ o ~ P a u l o ~ S a ̃ o ~ P a u l o ~(I C E S P), ~ S a ̃ o ~ P a u l o, ~ B r a z i l . ~}$

${ }^{c}$ Doctoral student, Department of Dental Materials and Prosthodontics, Aracatuba Dental School, Sao Paulo State University (UNESP), São Paulo, Brazil.

${ }^{d}$ Assistant, Dental Oncology Service, Institute of Cancer of São Paulo (ICESP), Sao Paulo, Brazil.

eProfessor, Department of Traumatology and Bucomaxillofacial Surgery, University of Sagrado Coração (USC), Sao Paulo, Brazil; and Coordinator,

Surgery Service of Brånermark Institute, São Paulo, Brazil.

fDepartment of Stomatology, School of Dentistry, University of Sao Paulo (USP), São Paulo, Brazil.

gDirector, Center of Oral Health Research, Omaha, Neb.; Professor, Periodontics, School of Dentistry, Omaha, Neb.; and Member and Past Chair,

Academic Partners, Center for Promoting Health and Health Equality, Creighton University, Omaha, Neb.

${ }^{h}$ Assistant Professor, Oral Diagnosis Department, Piracicaba Dental School, University of Campinas (UNICAMP), São Paulo, Brazil.

'Assistant Professor, Department of Prosthodontics and Periodontology, University of Campinas (UNICAMP), Piracicaba Dental School, São Paulo, Brazil.

'Section head, Maxillofacial Prosthodontics, Veteran's Affairs Nebraska-Western lowa Health Care System, Omaha, Neb.; and Special Associate Professor,

Department of Prosthodontics, Creighton University, Omaha, Neb.
} 


\section{Clinical Implications}

The most common reason for the replacement of facial prostheses is discoloration of the silicone material. Retouches may be used to delay the fabrication of new prostheses.

eyeglasses. ${ }^{2}$ However, neither of these methods provides satisfactory and durable retention, limiting the patients' activities and resulting in embarrassment when the prosthesis loses its retention during social interactions. 2,5,6

Craniofacial implants are a solution to maxillary defects, as they allow the use of more retentive methods and reduce the discoloration that results from conventional adhesives, yielding a more durable prosthesis. ${ }^{5,7}$ Moreover, the distress of a specialized and timeconsuming treatment is shortened, increasing the patient's overall well-being and acceptance of the rehabilitation treatment. ${ }^{5}$ Unfortunately, several clinical studies have reported that the survival of craniofacial implants varies depending on where they are placed. ${ }^{8-10}$ The current published data indicate predictable and high survival rates in the auricular region (approximately 95\%) and less favorable results at nasal $(71.4 \%$ to $100 \%)$ and orbital sites (27\% to $75 \%){ }^{8-10}$ Survival rate is not uniform across sites because of such factors as the quality and volume of the bone, hygiene, radiotherapy, hair-bearing tissue, skin mobility, and soft tissue thickness. ${ }^{8,10}$ While craniofacial implants are well covered by a wide range of published studies, studies that evaluate the clinical performance of facial prostheses are uncommon. The published clinical literature does not provide evidence-based information concerning this method of rehabilitation because it consists almost entirely of clinical reports, which primarily reflect personal experiences, and laboratory investigations. ${ }^{11-16}$

To date, silicone elastomers are the most commonly used to fabricate facial prostheses. ${ }^{1,13-16}$ However, the most common reason for the replacement of facial prostheses is the impairment of the properties of silicone elastomers, which is generally identified by the discoloration or failure of the material. ${ }^{6,13-22}$ This deterioration is caused primarily by exposure to weather conditions, human body secretions, and prosthesis maintenance procedures. ${ }^{1,6,12,23}$ Some studies have reported that the mean life span of a silicone-based facial prosthesis ranges from between 1.5 and 3 years, with only a small percentage of prostheses $(4.8 \%)$ lasting longer than 2 years. $6,12,24,25$ It is not surprising that the durability of facial prostheses remains a topic of discussion with the progressive increase in the number of patients with craniofacial defects. ${ }^{1}$ An urgent approach to improving this scenario is to provide more clinical evidence related to the life span of facial prostheses and to provide a complete overview of the common problems and complications for researchers, clinicians, and patients. This approach might help researchers to identify the factors that most affect the initiation of prosthesis replacement and the frequency of complications. ${ }^{6}$

Moreover, to the best of the authors' knowledge, few studies have assessed the need for surgical and prosthetic aftercare of implant-retained craniofacial prostheses. ${ }^{6,12}$ In addition, the current literature suggests that an objective clinical investigation that uses a descriptive analysis of prosthesis performance will help to optimize prosthetic treatment. Such a design provides realistic expectations about what can occur when patients with craniofacial defects receive facial prostheses retained conventionally or by using implants. ${ }^{14,24,25}$ Therefore, the purpose of this retrospective study was to estimate the type and rate of complications of facial prostheses and craniofacial implants through a retrospective analysis of patients over a follow-up period of up to 11 years; to analyze possible predictive variables for prostheses failure; and to highlight the results that can be expected for patients with craniofacial defects who receive implant or conventionally retained facial prostheses.

\section{MATERIAL AND METHODS}

Patients who had craniofacial defects, with or without craniofacial implants, and who were rehabilitated with silicone facial prostheses from January 2004 to March 2015 were evaluated in a retrospective record review. The records of a single Brazilian institution were screened (P-I Brånemark Institute, Bauru, São Paulo, Brazil). The institutional ethics committee of Piracicaba Dental School, University of Campinas (process number 1.594.462), granted approval for this study.

The patients' records were assessed, and data concerning sex, age, type of facial prosthesis, defect etiology, number of craniofacial implants, follow-up, prosthesis longevity, reasons for changing prostheses, retentive system, and prosthetic aftercare were collected. In the present study, facial prostheses were covered only by the Brazilian government health care system. All problems that did not lead to the fabrication of a new prosthesis, such as repairs of attachment systems, retouches, the fabrication of new bars, tightening of screws, changing of abutments, hygiene instructions, and skin reactions around the abutments connected to the craniofacial implants, were considered to be prosthetic aftercare. Problems that led to the fabrication of new prostheses and implant loss were considered to be failures. A data sheet was used to collect this information from every patient's records. Two specialized prosthetists (A.J.V.F. and T.B.B.) and 1 surgeon (H.N.F.) performed the rehabilitation of the patients evaluated in this study. 
Table 1. Summary of patient data according to defect type

\begin{tabular}{|c|c|c|c|c|c|c|}
\hline Defect Site & Participant, n & Age (y), Range/Mean & Sex & Defect Cause & $\begin{array}{l}\text { Implants } \\
\text { Placed, } n\end{array}$ & $\begin{array}{c}\text { Observation Period (mo), } \\
\text { Range/Mean }\end{array}$ \\
\hline Auricular & 10 & $10-40 / 24.5$ & 6 male; 4 female & 7 congenital; 3 trauma & 23 & $29-98 / 56.9$ \\
\hline Nasal & 8 & $25-76 / 63.5$ & 2 male; 6 female & 8 cancer & 12 & $13-57 / 44.4$ \\
\hline Orbital & 9 & $39-73 / 56.2$ & 5 male; 4 female & 7 cancer; 2 trauma & 20 & $31-79 / 58$ \\
\hline Total & 27 & $10-76 / 46.7$ & 13 male; 14 female & 15 cancer; 7 congenital; 5 trauma & 55 & $13-115 / 53.9$ \\
\hline
\end{tabular}

Prosthetic procedures were started 6 months after implant placement (2-stage procedure).$^{10}$ After implant exposure, the abutments were tightened using a $15 \mathrm{Ncm}$ torque control device, as recommended by the manufacturer (Brånemark System; Nobel Biocare). The periimplant skin was subjected to impression procedures only after a healing period of 2 to 4 weeks. An impression of the patient's face was made by using a polyvinyl siloxane. Casts were obtained with a Type IV colorless dental die stone (GC Fujirock; GC Corp). Sculpturing of the facial anatomy was performed with Chavant clay (Technovent; Factor II) on a layer of baseplate wax. . $^{8,24}$ The type of attachment systems used depended on the space available for prosthesis insertion. In the present study, bar clips and magnets were used (Magnetic Components; Factor II), and an acrylic resin framework was fabricated (Classico Autopolymerizing Acrylic Resin; Classico). A primer (A-330-G; Technovent, Factor II) was used to chemically enhance the bond of silicone elastomer to acrylic resins. Subsequently, the silicone elastomer (VST-50F Silicone Elastomer; Technovent, Factor II) was combined with pigments (FI; Functional Intrinsic II, Silicone Coloring System; Technovent, Factor II) and fiber flocking (H; Flocking; Technovent, Factor II), which were incorporated intrinsically, to match the patient's skin. Next, extrinsic coloration (KT199; Technovent, Factor II) was performed, if needed, with pigments associated with a transparent silicone (medical adhesive A; Technovent, Factor II). The patients were recalled for consultations every 6 months.

A standardized form was used to collect patient data, and new information regarding the craniofacial implants, prostheses, and peri-implant soft tissue were obtained at recall examinations after the prosthesis and patient had been subjected to clinical evaluation. The data collection was performed by 2 calibrated prosthodontists (A.J.V.F. and T.B.B.) with expertise in maxillofacial rehabilitation. The clinicians evaluated the acceptability of the facial prosthesis of each patient in terms of color alteration, quality of fit, satisfactory retention, hygiene, and integrity of the silicone material. Color alteration was defined as a decrease in the quality of the color match of the prosthesis to the underlying skin over time. Mechanical complications of the abutment and attachment components were also recorded by following the protocol described above. When both evaluators did not reach a consensus on each criterion for each patient, a third
Table 2. Frequency distribution of auricular, orbital, and nasal prostheses per patient and number of prostheses made for same patient over follow-up period

\begin{tabular}{lcccccr}
\hline & \multicolumn{7}{c}{ No. of Prostheses } \\
\cline { 2 - 7 } Prosthesis Type & $\mathbf{1}$ & $\mathbf{2}$ & $\mathbf{3}$ & $\mathbf{4}$ & $\mathbf{5}$ & $\mathbf{6}$ \\
\hline Auricular & 3 & 2 & 2 & 1 & - & 1 \\
\hline Nasal & 3 & 2 & 1 & 1 & 1 & - \\
\hline Orbital & 4 & 2 & 1 & 1 & - & - \\
\hline Total & 10 & 6 & 4 & 3 & 1 & 1 \\
\hline
\end{tabular}

prosthodontist (V.E.S.B.) was consulted to moderate discussion. The reasons for a new prosthesis were recorded. Failure was defined as the need for a new prosthesis caused by 1 or more of the factors that could impair prosthesis acceptability. When another fabrication of the prosthesis was necessary, the reasons were registered after the assessment of the prosthesis and patient by the evaluators.

The time variable was the number of days from the delivery of the facial silicone prosthesis to failure. Summary statistics were calculated for age, and frequencies were calculated for sex, type of prosthesis, source of defect, number of prostheses per patient, number of implants per patient, number of failures per patient, type of retention per patient, and reason for failures. All prostheses were included in a statistical analysis, which means the first delivered and all the other prostheses fabricated after each failure. Frailty survival modeling (simple random effects survival model) was used to test whether age, sex, type of prosthesis, source of defect, number of implants, and type of retention had a direct impact on the prosthetic failure rate. Statistical software (IBM SPSS Statistics v20.0; IBM Corp) was used for the analyses $(\alpha=.05)$.

\section{RESULTS}

This study evaluated a total of 27 patients (12 men, 15 women). The average age was 46.7 years (range 10 to 76 years). The characteristics of the patients evaluated in this study and the prosthetic results are displayed in Tables 1 , 2 , and 3. The most common type of prosthesis in this study was auricular. All of the auricular defects were congenital or resulted from trauma, in contrast to nasal and orbital defects resulting from cancer, trauma, or congenital origins. Fifty-five new prostheses were fabricated. In total, 84 prostheses were evaluated during the 
Table 3. Summary of prostheses characteristics, frequency distribution of reasons for prostheses aftercare, and failure types

\begin{tabular}{|c|c|c|c|}
\hline Prosthesis Type & Retention Type & No. of Prostheses Aftercare & No. of Failure Type \\
\hline Auricular & $\begin{array}{l}\text { - } 6 \text { bar clip } \\
\text { - } 1 \text { bar clip + adhesive } \\
4 \text { magnets }\end{array}$ & $\begin{array}{l}\text { - } 7 \text { retouches } \\
\text { - } 3 \text { new bar } \\
\text { - } 2 \text { changing of abutments } \\
\text { - } 1 \text { new clips } \\
\text { - } 1 \text { small rebonding of silicone to acrylic resin } \\
\text { - } 1 \text { reatment of exudate in peri-implant skin } \\
\text { reinement of acrylic resin framework }\end{array}$ & $\begin{array}{l}\text { - } 8 \text { color alteration } \\
\text { - } 4 \text { misfit } \\
\text { - } 1 \text { lack of retention } \\
\text { - } 1 \text { rupture of silicone } \\
\text { - } 1 \text { fracture of clips }\end{array}$ \\
\hline Nasal & $\begin{array}{l}\text { - } 4 \text { bar clip } \\
\text { - } 1 \text { bar clip + magnets } \\
\text { - } 2 \text { magnets } \\
\text { - } 2 \text { adhesive }\end{array}$ & $\begin{array}{l}\text { - } 7 \text { retouches } \\
\text { - } 1 \text { new clips }\end{array}$ & $\begin{array}{l}\text { - } 7 \text { color alteration } \\
\text { - } 3 \text { misfit } \\
\text { - } 1 \text { detachments of silicone to acrylic resin }\end{array}$ \\
\hline Orbital & $\begin{array}{l}\text { - } 1 \text { bar clip } \\
\text { - } 8 \text { magnets }\end{array}$ & $\begin{array}{l}\text { - } 7 \text { retouches } \\
\text { - } 1 \text { changing of abutments } \\
\text { - } 2 \text { retightening of abutment screw } \\
\text { - } 3 \text { treatment of exudate in peri-implant skin ( } 1 \text { implant loss) }\end{array}$ & $\begin{array}{l}\text { - } 7 \text { color alteration } \\
\text { - } 1 \text { misfit } \\
1 \text { rupture of silicone }\end{array}$ \\
\hline Total & $\begin{array}{l}\text { - } 11 \text { bar clip } \\
\text { - } 1 \text { bar clip + adhesive } \\
\text { - } 1 \text { bar clip + magnets } \\
\text { - } 2 \text { magnets }\end{array}$ & $\begin{array}{l}\text { - } 21 \text { retouches } \\
\text { - } 3 \text { new bar } \\
\text { - } 3 \text { changing of abutments } \\
\text { - } 1 \text { new clips } \\
\text { - } 4 \text { treatment rebonding of silicone to acrylic resin } \\
\text { - } 2 \text { retightening of abutment screw } \\
\text { - } 1 \text { reinforcement of acrylic resin framework }\end{array}$ & $\begin{array}{l}\text { - } 22 \text { color alteration } \\
\text { - } 8 \text { misfit } \\
\text { 1 lack of retention } \\
\text { - } 2 \text { detachment of silicone to acrylic resin } \\
\text { - } 1 \text { fracture of clips }\end{array}$ \\
\hline
\end{tabular}

follow-up period. Prosthetic follow-up after loading was more than 5 years for 8 patients, and no patient had less than 1 year of follow-up (mean follow-up of 53.9 months). Three patients died during follow-up because their cancer recurred. A total radiation dose could not be ascertained because participants who underwent rehabilitation at our institute were forwarded from private clinics and only medical consent was provided. Attempts were made to contact the relevant oncologists, but no response was obtained. All patients diagnosed with neoplasias were subjected to radiotherapy. Of the total study group, 1 patient had a conventionally retained prosthesis (adhesive).

Of the 84 prostheses, the most common reasons for replacement were color alteration $(n=23[27.4 \%])$, prosthesis misfit $(n=6[7.1 \%])$, detachment of silicone from the acrylic resin framework $(n=3[3.6 \%])$, and rupture of the silicone material $(\mathrm{n}=2$ [2.4\%]). Eleven patients had their prostheses colored with retouches. With regard to retentive methods, 23 participants $(85.2 \%)$ were provided with craniofacial implants, 2 participants retained their prostheses conventionally by using adhesives, and the other 2 participants used a combination of the 2 methods. Twelve individuals had their prostheses retained with magnets, 11 individuals had their prostheses retained with bar clips, 1 individual used a combination of bar clips and magnets, and 1 individual used bar clips and adhesive. In addition, fracture of clips was observed in 2 patients, while just 1 participant experienced complications with magnets.

Fifty-five craniofacial implants were placed, with most patients having 2 implants placed. Four craniofacial implants were placed in 1 participant who had bilateral auricular prostheses. These 2 auricular prostheses were evaluated as different prostheses because, even though
Table 4. Relative risks from survival modeling with random effects

\begin{tabular}{lccc}
\hline Variable & Relative Risk & $\mathbf{9 5 \% ~ C l}$ & $\boldsymbol{P}$ \\
\hline Sex & & & .968 \\
\hline Female & Reference & - & \\
\hline Male & 0.989 & $0.567-1.723$ & \\
\hline Age (y) & & & .224 \\
\hline$\leq 25$ & Reference & - & \\
\hline $26-40$ & 0.736 & $0.316-1.715$ & \\
\hline $41-60$ & 0.472 & $0.188-1.183$ & \\
\hline$\geq 61$ & 1.030 & $0.488-2.176$ & \\
\hline Type of prosthesis & & & .740 \\
\hline Ear & Reference & - & \\
\hline Nose & 1.238 & $0.639-2.395$ & \\
\hline Orbit & 1.006 & $0.505-2.003$ & \\
\hline Source of defect & & & .862 \\
\hline Cancer & Reference & - & \\
\hline Congenital & 1.112 & $0.589-2.111$ & \\
\hline Trauma & 0.899 & $0.402-2.011$ & \\
\hline Retention & & & .240 \\
\hline Adhesive & Reference & - & \\
\hline Bar clip & 0.308 & $0.085-1.111$ & \\
\hline Magnet & 0.288 & $0.079-1.051$ & \\
\hline Bar clip + magnet & 0.211 & $0.032-1.419$ & \\
\hline No. of implants & & & \\
\hline 0 & Reference & & \\
\hline 2 & 0.269 & $0.078-0.930$ & \\
\hline$\geq 3$ & 0.427 & $0.113-1.614$ & \\
\hline
\end{tabular}

Significance of $5 \%$.

they were used by the same patient, several factors led them to be categorized as independent. The mean dimensions of the craniofacial implants were $8.5 \times 3.75$ $\mathrm{mm}$. None of the craniofacial implants was loaded immediately. However, Table 4 shows that the number of implants approached statistical significance $(P=.06)$, with a reduced risk of failure when the patient had 2 implants 
compared with patients with zero implants and patients with more than 2 implants. Four patients developed peri-implant skin reactions, and 1 implant had to be removed because of frequent skin reactions. Moreover, the success rate for implant osseointegration was $98.2 \%$. No failures in implant osseointegration were observed after 60 months of follow-up. Screw loosening was observed in 2 participants. All participants received hygiene instructions for their prostheses and implants.

\section{DISCUSSION}

In the present study, the number of prostheses fabricated was more than twice the total number of patients. All facial prostheses were covered by the Brazilian health care system. However, in contrast with other countries, where people are covered by their insurance, our institute fabricated only 1 prosthesis per participant because the financial support provided by the government is not sufficient for all the individuals who seek prosthetic treatment. Therefore, the policy of our institute was to fabricate only 1 prosthesis per participant so that prosthodontists can rehabilitate a greater number of patients. Individuals were aware that other prostheses could be fabricated in private offices, but no participant reported the need.

Color alteration was the principal reason for new prostheses, which is consistent with previous studies. $6,17,24,25$ The degradation of the silicone material is caused primarily by exposure to weather, human body secretions, and prosthesis maintenance procedures. ${ }^{1,6,12,23}$ According to some laboratory studies, the energy of light irradiation during exposure to weather degrades the polymer network (a phenomenon called postpolymerization) by changing the number of polymer chains, as well as their cross-linking and angular arrangement in space. These changes affect light transmission through the silicone material. Moreover, the breakdown of the polymer chain bonds is also caused by the interaction of body secretions with the silicone polymer. ${ }^{15,16}$ Also, some investigations suggest that digital friction induces the detachment of the additives that are incorporated into the elastomer matrix, even when it is performed gently; unfortunately, hygiene procedures for facial prostheses are mandatory, so this cannot be avoided. ${ }^{18-20}$ This factor might also explain the excessive number of small repairs (retouches) that were observed in nearly all patients.

Figure 1 shows that most of the prostheses failed 1 to 3 years after clinical installation, indicating that the mean life span of the prostheses is similar to the values previously reported by other clinical studies. ${ }^{6,12,24,25}$ However, according to our experience with the participants evaluated in this study, the retouches performed at the patient recall visits prolonged the life of the silicone facial prostheses. Such a strategy is worthwhile only with short-term recalls (maximum of 6 months) because once the silicone has been contaminated, color touch-ups do not last.

Misfit of the prostheses was also among the most frequent patient concerns and was also reported in a previously published study that prospectively evaluated 76 patients. ${ }^{4}$ The same mechanisms that cause discoloration may also affect the dimensions of the silicone elastomer because changes in the polymer network result in a shrunken material with reduced elasticity and increased hardness. ${ }^{18}$ In the present study, a poor fit was also observed when young patients grew or when patients lost weight because of cancer activity or depression. Thus, the data reported here, in combination with the published clinical studies, will help clinicians to monitor their patients with regard to these complications, as an estimated survival time based on clinical data enables clinicians to have realistic expectations regarding prostheses $^{24}$ and to work in a multidisciplinary team (including psychiatrists and psychologists).

In the present study, the youngest mean age was observed in patients with auricular prostheses, which was the type of prosthesis with the highest incidence of misfits. Auricular prostheses would be recommended for further clinical studies to monitor the dimensions and morphology of the defects in order to identify how changes in the fit of the prosthesis occur over time. That way, any changes that result from defects that evolve over time can be identified instead of attributing the misfit to only the silicone material; auricular prostheses are generally associated with congenital or traumatic factors, in contrast with nasal and orbital defects, which are generally associated with cancer.

Another common complication and reason for constant repairs during the prosthesis aftercare period is the detachment of silicone from the acrylic resin framework. ${ }^{24}$ This effect is explained by the differences in the chemical structures of these 2 materials (dimethyl siloxane polymers from silicone and polymethyl methacrylate from the resin). ${ }^{13,14}$ Some laboratory studies reported that the highest bond strength is achieved and maintained after aging when primers are used. Thus, we used 1 primer to improve the bonding between the 2 materials for all patients with implant-retained facial prostheses. These agents contain an organic solvent and an adhesive agent that reacts with both silicone and resin materials by activating the surfaces through etching or promoting hydrogen bonding and covalent coupling; this increases the wettability of the substrate by impregnating the surface layer with the polymeric ingredients. ${ }^{13,14}$ However, this chemical interaction must still be improved, as the detachment of silicone from the acrylic resin framework was also observed in some of our patients. 

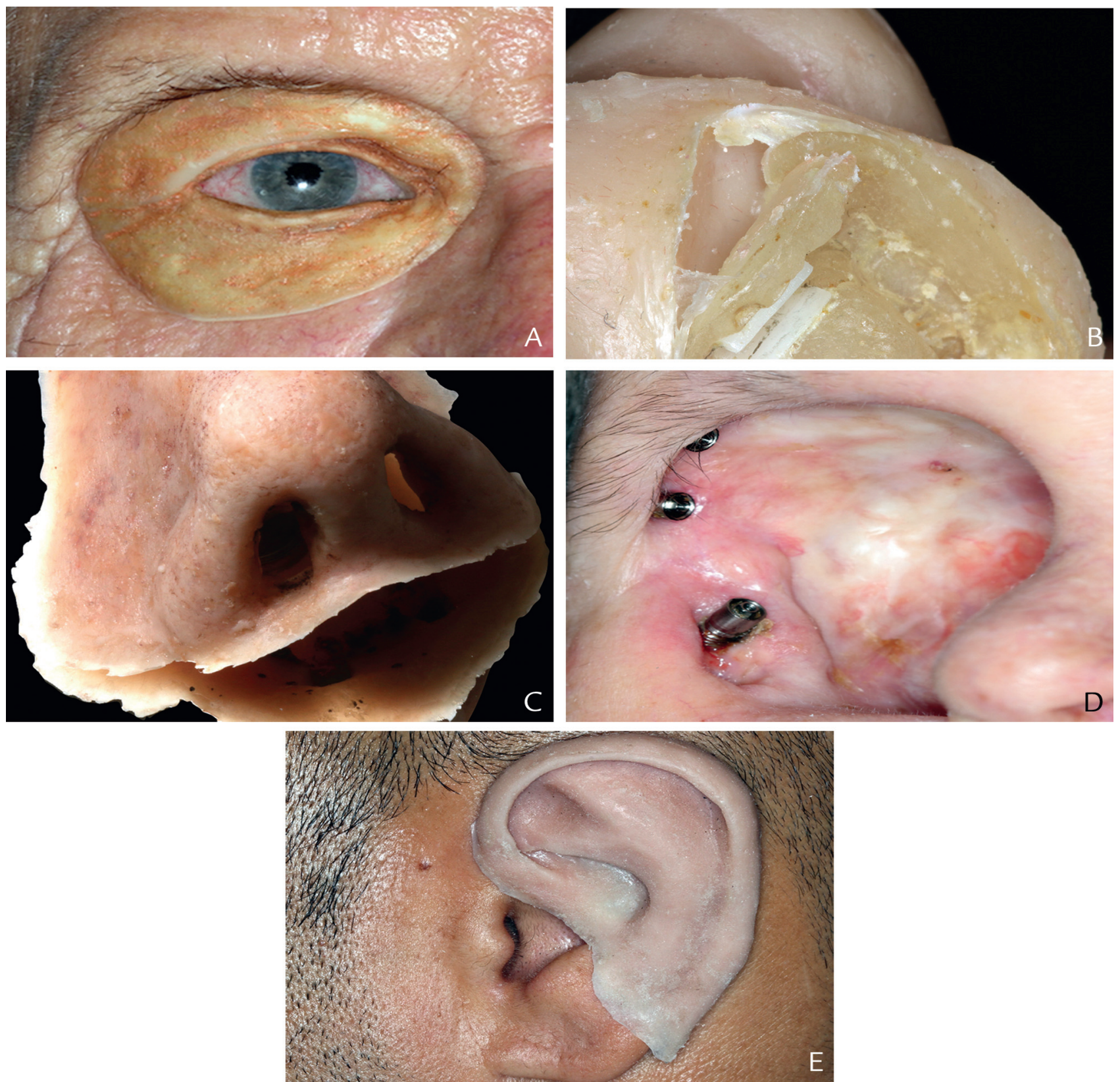

Figure 1. Illustration of complications among evaluated patients. A, Silicone discoloration causing mismatch with patient's skin. B, Debonding of silicone from acrylic resin infrastructure. C, Black stains on both inner and external silicone surfaces. D, Peri-implant skin reactions. E, Misfit between prosthesis and skin.

In addition, a less common complication was observed in this study: black stains on the surface of the prostheses and odors. These problems occur because silicone surfaces are susceptible to microorganisms that penetrate the pores of the silicone and are not removed by washing the prostheses. ${ }^{6,21}$

In contrast with another study, ${ }^{21}$ loosening of the bar screws was a complication noted in orbital prostheses. As a result, this type of prosthesis should always be associated with magnets when cost and prosthetic space allow. This complication is more frequently associated with clip retentions. ${ }^{21}$
Skin reactions around abutments were the most common complication observed in patients with craniofacial implants. This complication might occur because the peri-implant skin is a thin and immobile tissue, which results in fewer peri-implant tissue complications. ${ }^{8,6}$ In this study, only 1 implant had to be removed because of persistent tissue reactions rather than because of the loss of osseointegration. However, satisfactory survival rates are not common in the majority of published studies. ${ }^{8-10}$ Such high success rates were probably observed because this study was a pilot study and data 
could only be obtained from a small number of patients, and because some patients had a shorter follow-up period than others. Furthermore, skin reactions were most commonly associated with a lack of hygiene, and, as also observed in the another study, ${ }^{8}$ the problem was generally solved when this parameter was improved. Some patients in this study had their abutments changed or a new bar fabricated to facilitate hygiene maintenance, which may be a useful strategy for clinicians when adaptation time is not sufficient to solve the problem. Interestingly, the simple random effects survival model for implant failure did not reveal significant differences, but the $P$ value $(P=.06)$ approached statistical significance. To the best of the authors' knowledge, no study has evaluated the optimal number of craniofacial implants, and such a result might guide maxillofacial prosthodontists when planning rehabilitation with implant-retained facial prostheses.

The present study had some limitations. First, the study included a small number of participants from only 1 center. Second, some of the patients had a shorter follow-up period than others, and age ranged considerably. Younger individuals may have different patterns of wear than the older population. Further investigations should take a number of factors into consideration: a larger cohort size, evaluation of different predictive variables to identify the factors that result in prosthetic failure (such as the size of the prosthesis), different craniofacial rehabilitation centers (because populations in different geographic locations provide a more relevant clinical scenario), and longer follow-up periods with participants of similar age are necessary to obtain a more relevant clinical scenario.

\section{CONCLUSIONS}

Within the limitations of this retrospective pilot study, the following conclusions were drawn:

1. Color alteration is the principal reason for prosthesis replacement.

2. Prosthesis misfit, the detachment of silicone from the acrylic resin framework, and skin reactions around abutments were observed frequently.

3. Retouches can be performed to avoid the fabrication of new prostheses.

4. Well-designed studies are necessary to identify additional relevant complications and different predictive variables that result in prosthetic failure.

\section{REFERENCES}

1. Ariani N, Visser A, van Oort RP, Kusdhany L, Rahardjo TB, Krom BP, et al Current state of craniofacial prosthetic rehabilitation. Int J Prosthodont 2013;26:57-67.

2. Nemli SK, Aydin C, Yilmaz H, Bal BT, Arici YK. Quality of life of patients with implant-retained maxillofacial prostheses: a prospective and retrospective study. J Prosthet Dent 2013:109:44-52.
3. Chang TL, Garrett N, Roumanas E, Beumer J 3rd. Treatment satisfaction with facial prostheses. J Prosthet Dent 2005:94:275-80.

4. Markt JC, Lemon JC. Extraoral maxillofacial prosthetic rehabilitation at the MD Anderson Cancer Center: a survey of patient attitudes and opinions. J Prosthet Dent 2001:85:608-13.

5. Schoen PJ, Raghoebar GM, van Oort RP, Reintsema H, van der Laan BF, Burlage FR, et al. Treatment outcome of bone-anchored craniofacial prostheses after tumor surgery. Cancer 2001;92:3045-50.

6. Visser A, Raghoebar GM, van Oort RP, Vissink A. Fate of implant-retained craniofacial prostheses: life span and aftercare. Int J Oral Maxillofac Implants 2008;23:89-98.

7. Tolianic JA, Eckert SE, Roumanas E, Beumer J, Huryn JM, Zlotolow IM, et al. Osseointegrated craniofacial implants in the rehabilitation of orbital defects: an update of a retrospective experience in the United States. J Prosthet Dent 2005:94:177-82

8. Karakoca S, Aydin C, Yilmaz H, Bal BT. Survival rates and periimplant soft tissue evaluation of extraoral implants over a mean follow-up period of three years. J Prosthet Dent 2008;100:458-64.

9. Roumanas ED, Freymiller EG, Chang TL, Aghaloo T, Beumer J 3rd. Implant retained prostheses for facial defects: an up to 14-year follow-up repor on the survival rates of implants at UCLA. Int J Prosthodont 2002;15: 325-32.

10. Curi MM, Oliveira MF, Molina G, Cardoso CL, Oliveira Lde G, Brånemark PI, et al. Extraoral implants in the rehabilitation of craniofacial defects: implant and prosthesis survival rates and peri-implant soft tissue evaluation. J Oral Maxillofac Surg 2012;70:1551-7.

11. de Carvalho BM, Freitas-Pontes KM, de Negreiros WA, Verde MA. Single stage osseointegrated implants for nasal prosthodontic rehabilitation: a clinical report. J Prosthet Dent 2015:114:293-6.

12. Watson RM, Coward TJ, Forman GH. Results of treatment of 20 patients with implant-retained auricular prostheses. Int J Oral Maxillofac Implants 1995;10:445-9.

13. Hatamleh MM, Watts DC. Bonding of maxillofacial silicone elastomers to an acrylic substrate. Dent Mater 2010;26:387-95.

14. Hatamleh MM, Watts DC. Mechanical properties and bonding of maxillofacial silicone elastomers. Dent Mater 2010;26:185-91.

15. Al-Harbi FA, Ayad NM, Saber MA, ArRejaie AS, Morgano SM. Mechanical behavior and color change of facial prosthetic elastomers after outdoor weathering in a hot and humid climate. J Prosthet Dent 2015;113:146-51.

16. Hatamleh MM, Watts DC. Porosity and color of maxillofacial silicone elastomer. J Prosthodont 2011;20:60-6.

17. Hooper SM, Westcott T, Evans PL, Bocca AP, Jagger DC. Implant-supported facial prostheses provided by a maxillofacial unit in a UK regional hospital: longevity and patient opinions. J Prosthodont 2005;14:32-8.

18. Eleni PN, Perivoliotis D, Dragatogiannis DA, Krokida MK, Polyzois GL, Charitidis CA, et al. Tensile and microindentation properties of maxillofacial elastomers after different disinfecting procedures. J Mech Behav Biomed Mater 2013;28:147-55.

19. Eleni PN, Krokida MK, Polyzois GL, Gettleman L. Effect of different disinfecting procedures on the hardness and color stability of two maxillofacial elastomers over time. J Appl Oral Sci 2013;21:278-83.

20. Eleni PN, Krokida MK, Polyzois GL, Gettleman L. Dynamic mechanical thermal analysis of maxillofacial prosthetic elastomers: the effect of different disinfecting aging procedures. J Craniofac Surg 2014;25:e251-5.

21. Polyzois GL, Tarantili PA, Frangou MJ, Andreopoulos AG. Physical properties of a silicone prosthetic elastomer stored in simulated skin secretions. J Prosthet Dent 2000;83:572-7.

22. Abu-Serriah MM, McGowan DA, Moos KF, Bagg J. Outcome of extra-oral craniofacial endosseous implants. Br J Oral Maxillofac Surg 2001;39:269-75.

23. Leonardi A, Buonaccorsi S, Pellacchia V, Moricca LM, Indrizzi E, Fini G. Maxillofacial prosthetic rehabilitation using extraoral implants. J Craniofac Surg 2008;19:398-405.

24. Karakoca S, Aydin C, Yilmaz H, Bal BT. Retrospective study of treatment outcomes with implant-retained extraoral prostheses: survival rates and prosthetic complications. J Prosthet Dent 2010;103:118-26.

25. Jani RM, Schaaf NG. An evaluation of facial prostheses. J Prosthet Dent 1978;39:546-50.

Corresponding author:

Dr Thais Bianca Brandão

Dental Oncology Service, Institute of Cancer of São Paulo (ICESP)

Faculty of Medicine, University of São Paulo

Arnaldo Ave, 251, Cerqueira Cesar

Sao Paulo, 01246-000

BRAZIL

Email: thais.brandao@icesp.org.br

\section{Acknowledgments}

The authors thank Per-Ingvar Brånemark (in memoriam) for all his devotion and contributions to patients with craniofacial defects.

Copyright () 2016 by the Editorial Council for The Journal of Prosthetic Dentistry. 